\title{
Whither Neuropathology?
}

Can. J. Neurol. Sci. 2003; 30: 299-301

The Royal College of Physicians and Surgeons of Canada is contemplating abolishing neuropathology as a primary specialty. This includes the examination in neuropathology. As part of a housecleaning of small, unneeded specialties, neuropathology is targeted. It has also been mentioned that it is costly to examine but one or two candidates in any given year. The idea of holding the neuropathology board examination in conjunction with the annual meeting of the Canadian Association of Neuropathologists (CANP) meeting seems not to have been entertained. I am sure that Canadian Neuropathologists would be willing to move the CANP meeting, if that is what it would take to save neuropathology. But no one seems to have asked. The targeting of neuropathology for elimination as a primary specialty is certain to demote quality since the days when those with foresight crafted the specialty according to need.

Dr. Harry Botterell was one of those individuals, a prominent neurosurgeon from Toronto, who approached the college outlining the need for neuropathology in neurosurgery. Since Drs. N. Barry Rewcastle, David Robertson, Clarissa ("Laurie") Dolman and Gordon Mathieson sat the first exam in neuropathology in Canada in 1968 (given by the great tumor neuropathologist Dr. Lucien J. Rubinstein), there has been a proliferation of successful candidates in neuropathology, who have spread over most of Canada to provide coverage for the reading of biopsies of the brain, spinal cord, nerve and muscle. No one seriously thinks that more excellence, or more candidates, or more Canadian-based publications in neuropathology, would be obtained by abolishing this system, which has worked well for over 35 years in Canada.

\section{SEEN MANY WAYS, NEUROPATHOLOGY IS A FREE-STANDING SPECIALTY:}

\section{Annual neuropathology meetings are thriving.}

The annual meeting of the Canadian Association of Neuropathologists has been thriving, and shows no signs of slowing down. The International Society of Neuropathology meeting attracts 1000 people worldwide, and is vigorous after many decades. Non-specialties die in and of themselves. They should not be killed or actively removed if they are viable and working to serve the public and the medical community. The fact that U.S. delegates come in large numbers to the Canadian Association of Neuropathologists annual meeting (a reverse of the usual) speaks for itself. We must be doing something right in Canada. We attract people because we have a distinct specialty doing neuropathology. Canada is perceived by other countries as a leader country in neuropathology.

2. Neuropathology is a distinct, advancing, primary body of knowledge.

Although intersecting with anatomical pathology, neurosurgery, neurology, neuroradiology and basic neuroscience, it is not a subset of any of these. Neuropathology has been called a lynchpin, because of its small size yet its necessary, supporting role of so many areas. So many interfaces exist (see Venn diagram.). The existence of the 7th edition of Greenfield's Neuropathology indicates a viable, distinct body of knowledge. There are over 1000 neurological diseases. Most organ systems have one tenth of that. New neurological diseases are continuously recognized. Who will recognize the neuropathology of new diseases (eg. neurocytoma, AIDS in the brain, domoic acid [toxic mussel poisoning] intoxication or dysembryoplastic neuroepithelial tumor), if not neuropathologists? Who will make advances in the field? When Gordon Mathieson and Canadian neurologist/ neuroscientist Tony Hakim got together and looked for the cause of dementia in Parkinson's disease, they wrote a paper ${ }^{1}$ that has been cited 300 times (Institute for Scientific Information).

"The existence of a specialty is justified only so long as it makes such rapid progress that the larger group from which it has split off cannot keep pace with it."

- Harvey Cushing

\section{Neuropathology is not a branch of anatomical pathology.}

In the unfortunate turf war between neuropathology and anatomical pathology, neuropathology is often tacitly assumed to be a branch of anatomical pathology. The brain is assumed to be just another tissue, just another organ. Nothing could be further from the truth. Without hyperbole, the brain is the most

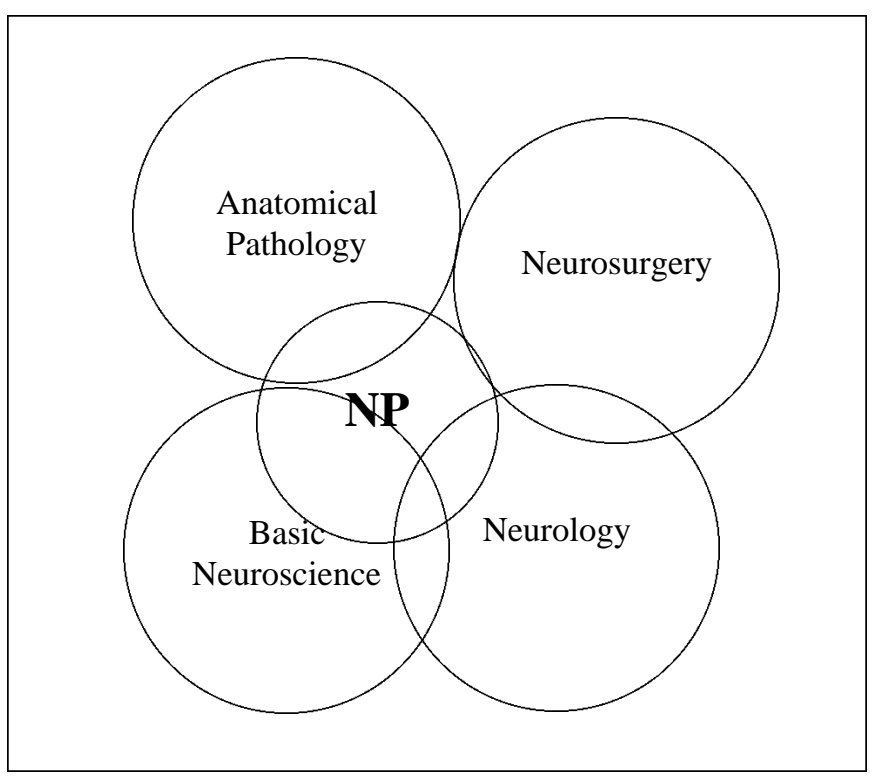


complicated structure in the universe. This includes the CPU in our most advanced computers. To drive this point home, consider an operation on your own brain. There are specialists called neurosurgeons and surgeons, and most of us would prefer a neurosurgeon. No one is talking about folding neurosurgery back into surgery, even though we have a big fish/little fish situation there, too. This would be too obviously dangerous and retrogressive, too publicly unwise, since direct patient contact is involved. Neurosurgeons have a certain feel that neuropathology should exist, as they know things happen in the brain that happen nowhere else. Neurosurgeons know well the dangers of considering the brain as just another organ. They know that lesion location determines the likely nature of the lesion. This happens in no other organ as it does in the brain. Lose your right or your left lung or kidney, and the consequences are identical. This raises the whole spectre of neuroanatomy as a necessary foundation of neuropathology. Neuroanatomy determines both the likely nature of the lesion and its clinical presentation. This is a fundamental cornerstone of our communication with clinicians. Not only this, but the sheer volume of information in neuroanatomy is in no way a subset of anatomical pathology. In these ways, the practice of neuropathology is fundamentally different from anatomical pathology. And then there are those more than 1000 diseases of the nervous system. Again, not a branch of anatomical pathology. Teased fibre examination of nerve and neuropathological special stains need overseeing by neuropathologists. Quality control of such requires a dedicated neuropathologist and a dedicated neuropathology laboratory, not a branch of anatomical pathology. The knowledge base, mode of thinking and daily practice of neuropathology thus make neuropathology anything but a subspecialty of anatomical pathology.

\section{Risks of abolishing neuropathology.}

Examination of the brain is one of the most intricate and integrative procedures performed in medicine. A well-trained anatomical pathologist is not necessarily competent to deal with neuropathology. It is risky for the individual anatomical pathologist forced into practicing neuropathology against his/her better judgment. Quality will go down if neuropathology is abolished, especially with abolition of the fellowship exams. Errors and lawsuits can only go up. Many kinds of tumors exist only in the brain. Accurate diagnosis is essential (eg. ganglioglioma vs. astrocytoma, neurocytoma vs. oligodendroglioma). Other countries not having neuropathology are constantly faced with the double dilemma of forcing neuropathologists to do anatomical pathology and forcing anatomical pathologists to do neuropathology. This makes clinicians uncomfortable, and with good reason. Both are dangerous situations for all concerned (the practicing pathologist, the patient, and the clinician). Creating this danger, and possible litigation, cannot be condoned.

\section{Career paths must remain open.}

Neuropathology training can be entered by multiple initial routes. Usually a change of heart occurs in a career path: heart and soul, as well as knowledge, must be applied in any specialty, to practice effectively. Thinking that the universal entry point to become a neuropathologist should be made via anatomic pathology is folly, since a major portion of neuropathologists have had entry points via neurology or neurosurgery. Direct entry into neuropathology must also remain a viable career route. If a four year program of anatomical pathology is imposed on neuropathology, and two years of neuropathology are merely tagged on at the end, neuropathology will surely die. Who will then be responsible for this? We must accept that neuropathology is a distinct body of knowledge and practice, with multiple entry points that need to be maintained. It is quite fragile, with only eight new neuropathologists board-certified by the Royal College 1997-2002. We cannot tinker with this, or we will reduce the flow of new neuropathologists. Telling neurologists or neurosurgeons in training that if they wish to switch to neuropathology they must first do anatomical pathology, is a virtual kiss-of-death for neuropathology. It guarantees neuropathology will wither.

\section{Access to pathology by clinicians.}

Dedication to the specialty of neuropathology makes for free access to neuropathologists by clinicians. A year of clinical training should be built into neuropathology, to ensure the common language of clinical neuroscience is learned by the neuropathology resident. The common parlance makes us neuropathologists more freely available intellectually to our clinical colleagues whom we serve. We better understand the problems we are helping to solve. These matters cannot be left to chance. They must be built into the neuropathology training program. The clinical year was included in bygone years, but has since been deleted. The founders may have had it right the first time.

\section{Medico-legal considerations.}

With due respect to the medico-legal system, mistakes are made when neuropathology is not involved. A common error is attribution of death to the wrong cause, trauma as cause of death being spuriously ruled out. This error occurs especially if there is a delay between trauma and death. Only two examples will be given here. In one case, an 18-year-old helmeted cyclist was struck by a car and rolled up the windshield of the car. He died seven weeks later of a cerebellar infarct and brainstem compression, but the motor vehicle accident was thought not to have played a role. However, if relations with neuropathology are good, an "in course correction" can be made. In this case, John Butt, Calgary's Chief Medical Examiner at the time, and I examined the vertebral arteries, and found old arterial dissection. The driver was charged with causing the death. The case was published, ${ }^{2}$ with the instructive title "Delayed symptoms and death following minor head trauma with occult vertebral artery injury". We learn that lateral rotation of the head while rolling up the hood of a car can, like chiropractic manipulation, lead to vertebral artery dissection, and we learn also that delayed death can occur after minor head trauma. A second case involved the widely publicized August 13, 2000 robbery/murder in Regina, where the assailants beat an elderly couple into coma. The husband died on the spot of head trauma. The wife, found still breathing two days later, awoke from her coma after two weeks. She had cardiac arrhythmias while in hospital. Two weeks later (four weeks after the head beating) she died, shortly after being informed that her husband was dead. Autopsy revealed no coronary occlusion, and initially, the case was perplexing. I examined the referred brain, and concluded that the massive, 
widespread contusional injury and internal shearing, including the region of the insula, led to the cardiac arrhythmias and that this was a neurocardiac death. There is extensive basic neurocardiologic literature explaining the patient's clinical course and death, which the courts and the non-neuropathologic medical community were unaware of. The individuals were charged with murder. For such co-operation with the medicolegal system to exist, there must first be neuropathology to cooperate with.

\section{CAN NEUROPATHOLOGY BE ABOLISHED AND MADE A BRANCH OF ANATOMICAL PATHOLOGY?}

Administratively, it certainly can, but at what price? The effects would not be immediate, but insidious over the coming years. And what are we fixing here? This small specialty has found a niche existence, with so many interactions medicolegal, neurosurgical, neurological and basic neuroscience. Abolishing it as a primary specialty serves no purpose other than social engineering of doctors, a phenomenon rampant in medicine nowadays. If abolition is part of the turf war between anatomical pathology and neuropathology, then it is an especially disgraceful battle. If abolishing neuropathology is part of cost control of the Royal College examinations, then let the examinations take place in conjunction with the CANP Annual Meeting, where candidates and examiners are already present in the same hotel. Costs then incurred would then be minimal, and involve no more than an extra day or two of stayover. It would be sad if Canada removes neuropathology as a specialty because we cannot pay for the exam. There are solutions. But what precisely is the problem with having neuropathology as a primary specialty? It seems to have served Canada well, including its medical, legal, scientific and patient communities, for many decades. Neuropathology should not be swept away into the dustbin of medical history of Canada. This would be a retrogressive step.

Roland Auer Calgary, $A B$

\section{REFERENCES}

1. Hakim AM, Mathieson G. Dementia in Parkinson's disease: a neuropathologic study. Neurology 1979;29:1209-1214.

2. Auer RN, Krcek J, Butt JC. Delayed symptoms and death following minor head trauma with occult vertebral artery injury. J Neurol Neurosurg Psychiatry 1994;57:500-502.

\section{Textbooks}

The following are well used standard neuropathology textbooks authored by Canadian Neuropathologists in the last 20 years.

1. Dolman CL. Ultrastructure of Brain Tumors and Biopsies: A Diagnostic Atlas. New York: Prager 1984.

2. Davis RL, Robertson DM. Textbook of Neuropathology. 2nd Ed. Baltimore: Williams \& Wilkins.

3. Norman MG, et al. Congenital Malformations of the Brain: Pathological, Embryological, Clinical, Radiological and Genetic Aspects. New York: Oxford University Press, 1995.

4. Midroni G, Bilbao JM. Biopsy Diagnosis of Peripheral Neuropathy. Boston: Butterworth/Heineman 1995.

5. Carpenter S, Karpati G. Pathology of Skeletal Muscle. New York: Churchill \& Livingstone, 2nd Edition, 2001. 